\title{
Oberflächennahe Geothermie: Regelungsbedarf zur Berücksichtigung ökologischer and technischer Aspekte?
}

\author{
Hilke Würdemann · Philipp Blum
}

Eingang des Beitrages: 18.3.2011 / Online veröffentlicht: 3.5.2011

(C) Springer-Verlag 2011

Der rapide Anstieg der Erdöl- und Erdgaspreise und die dringende Notwendigkeit zur Minderung der $\mathrm{CO}_{2}$-Emissionen beschleunigen die Entwicklung von Verfahren zur effizienteren Energienutzung und zur Gewinnung erneuerbarer Energie. Aus diesem Grund werden auch die geothermische Wärme- und Stromerzeugung sowie die Wärme- und Kältespeicherung weiter zunehmen. Geothermische Anlagen führen je nach Art der Nutzung zu einer Abkühlung oder Erwärmung des Untergrundes. Obwohl die Temperatur eine Schlüsselgröße für geochemische und biologische Prozesse ist, wurden die technischen und ökologischen Aspekte der geothermischen Nutzung im Untergrund bisher kaum wissenschaftlich untersucht. Die Auswirkungen der Temperaturänderung auf die natürlichen Lebensgemeinschaften im Untergrund und der Einfluss der Geochemie der Wässer sind noch weitgehend unbekannt. Im Rahmen dieses Themenheftes werden Forschungsergebnisse in Bezug auf die Veränderungen im Ökosystem durch die geothermische Nutzung des Untergrundes und die Bedeutung mikrobieller Stoffwechselprozesse für den Anlagenbetrieb vorgestellt. Der Beitrag von Hähnlein et al. gibt einen Überblick zu den rechtlichen Rahmenbedingungen und den Konsequenzen für die praktische Umsetzung in Deutschland. Die Studie zeigt wie heterogen diese nationalen als auch internatio-

\footnotetext{
H. Würdemann

Deutsches GeoForschungsZentrum GFZ, Internationales Geothermiezentrum, Telegrafenberg, 14473 Potsdam, Deutschland

E-Mail: wuerdemann@gfz-potsdam.de

P. Blum ( $\varangle)$

Karlsruher Institut für Technologie (KIT), Institut für Angewandte Geowissenschaften (AGW), Kaiserstraße 12, 76131 Karlsruhe, Deutschland E-Mail: philipp.blum@kit.edu
}

nalen Regelungen in Bezug auf die oberflächennahe Geothermie momentan sind und belegt den dringenden Handlungsbedarf.

In der Studie von Brielmann et al. wird der Einfluss von Temperaturänderungen in Labor- und Felduntersuchungen analysiert und deren Bedeutung für das Ökosystem Grundwasser diskutiert. Lerm et al. und Alawi et al. untersuchen die technischen Auswirkungen mikrobiologischer Stoffwechselprozesse auf den Anlagenbetrieb anhand von Beispielen aus der Praxis. Lerm et al. zeigen, dass einzelne Mikroorganismen aufgrund ihrer hohen Sensitivität und ihrer über die Zeit integrierenden Funktion als Indikatororganismen für geochemische Veränderungen und deren Auswirkungen auf Filterstandzeit und Injektivität dienen können. Alawi et al. beobachteten eine hohe Diversität sulfatreduzierender Mikroorganismen nach dem Wärmetauscher einer tiefen geothermischen Anlage, die auf eine mögliche Bedeutung dieser Organismen für Korrosionsprozesse im Bereich der kalten Seite hinweisen.

Der Beitrag von Stober \& Jodocy befasst sich mit dem geothermischen Potenzial im südlichen Oberrheingraben. Reflexionsseismische Daten wurden zur Abschätzung der Produktivität potenzieller Nutzhorizonte herangezogen. Weil die in diesem Becken zu erwartenden Wässer hochmineralisiert sind, müssen im Zuge einer geothermischen Nutzung geochemische und mikrobiologische Aspekte hier ebenfalls berücksichtigt werden, um die Wahrscheinlichkeit des Auftretens von injektivitätsmindernden Ausfällungen gering zu halten. Fundierte wissenschaftliche Untersuchungen zur Veränderlichkeit des Grundwasserökosystems und die Bewertung der ökologischen und technischen Auswirkungen im Gesamtkontext sind deshalb eine wesentliche Voraussetzung für die längst überfällige Festlegung wissenschaftlich begründeter Temperaturgrenzwerte für die oberflächennahe geothermische Nutzung des Untergrundes. 
Die zunehmende Nutzung verstärkt die Notwendigkeit zu einem verbesserten Prozessverständnis und zur Weiterentwicklung regulatorischer und empfehlender Vorgaben. Mit diesem Themenheft wollen wir einen ersten Schritt in Richtung dieser gesamtheitlichen Betrachtung unternehmen.
Wir hoffen, dass dieses Themenheft auf Ihr Interesse stößt, zu weiteren wissenschaftlichen Studien inspiriert und zu einer weiterführenden Diskussion zwischen den planenden Ingenieurbüros, den Betreibern und den Genehmigungsbehörden führt. 